\title{
Summary of cerebrospinal fluid routine parameters in neurodegenerative diseases
}

\author{
Sarah Jesse • Johannes Brettschneider • Sigurd D. Süssmuth • Bernhard G. Landwehrmeyer • \\ Christine A. F. von Arnim • Albert C. Ludolph - Hayrettin Tumani • \\ Markus Otto
}

Received: 23 September 2010/Revised: 14 November 2010/ Accepted: 9 December 2010/Published online: 25 December 2010

(C) The Author(s) 2010. This article is published with open access at Springerlink.com

\begin{abstract}
In neurodegenerative diseases, cerebrospinal fluid analysis (CSF) is predominantly performed to exclude inflammatory diseases and to perform a risk assessment in dementive disorders by measurement of tau proteins and amyloid beta peptides. However, large scale data on basic findings of CSF routine parameters are generally lacking. The objective of the study was to define a normal reference spectrum of routine CSF parameters in neurodegenerative diseases. Routine CSF parameters (white cell count, lactate and albumin concentrations, CSF/serum quotients of albumin $\left(Q_{\mathrm{alb}}\right)$, IgG, IgA, IgM, and oligoclonal IgG bands
\end{abstract}

Electronic supplementary material The online version of this article (doi:10.1007/s00415-010-5876-x) contains supplementary material, which is available to authorized users.

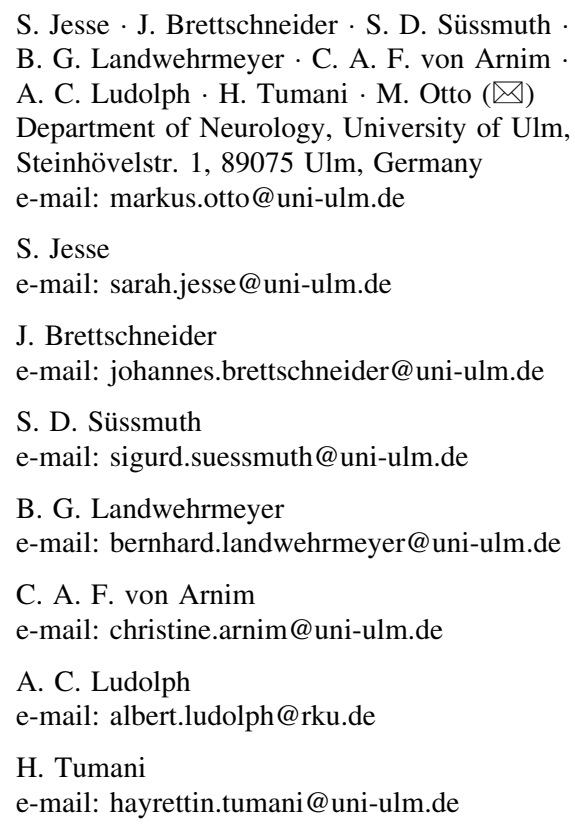

$(\mathrm{OCB}))$ were retrospectively analyzed in an academic research setting. A total of 765 patients (Alzheimer's disease (AD), Parkinson's disease (PD), Parkinson's disease dementia (PDD), vascular dementia (VD), frontotemporal lobar degeneration (FTLD), progressive supranuclear palsy (PSP), multisystem atrophy (MSA), motor neuron diseases (MND), spinocerebellar ataxia (SCA), Huntington's disease (HD)) and non-demented control groups including a group of patients with muscular disorders (MD). The main outcome measures included statistical analyses of routine CSF parameters. Mildly elevated $Q_{\text {alb }}$ were found in a small percentage of nearly all subgroups and in a higher proportion of patients with PSP, MSA, VD, PDD, and MND. With the exception of 1 MND patient, no intrathecal Ig synthesis was observed. Isolated OCBs in CSF were sometimes found in patients with neurodegenerative diseases without elevated cell counts; lactate levels were always normal. A slightly elevated $Q_{\text {alb }}$ was observed in a subgroup of patients with neurodegenerative diseases and does not exclude the diagnosis. Extensive elevation of routine parameters is not characteristic and should encourage a re-evaluation of the clinical diagnosis.

Keywords Cerebrospinal fluid - Neurochemical investigation - Standard routine parameters .

Neurodegenerative disorders · CSF flow

\section{Introduction}

Neurodegenerative disorders represent a major health problem that negatively affect the quality of life in our aging society [23]. Until the early 1990s, neurochemical laboratory diagnosis of neurodegenerative disorders predominantly concentrated on the exclusion of other 
neurological diseases, especially those of inflammatory origin. Nowadays, CSF analyses are also performed to measure biomarkers associated with specific pathomechanisms of the underlying neurodegenerative disease [33]. Nevertheless-prior to the application of more specific CSF parameters-a routine work-up of basic CSF findings should be performed [27]. So far, a large scale systematic evaluation of routine CSF parameters including white cell count, lactate concentration, CSF to serum ratios of albu$\min \left(Q_{\mathrm{alb}}\right)$, and immunoglobulins IgG, IgA, and IgM does not exist. Furthermore, various studies on mostly smaller cohorts of patients reported conflicting evidence with regard to basic CSF findings. For example, Fröhlich et al. [8] detected a normal blood-CSF barrier function in Alzheimer's disease (AD) and no evidence of an intrathecal synthesis of IgG. In contrast, Hampel et al. isolated oligoclonal bands (OCB) in CSF in up to $20 \%$ of AD patients, indicating a humoral immune response within the central nervous system (CNS), accompanied by a blood-CSF barrier dysfunction present in $16 \%$ of the cases. Likewise, Zimmermann et al. [37] found intrathecal IgG synthesis in some of their $\mathrm{AD}$ patients without identifying a satisfying reason for the intrathecal humoral response. These findings were confirmed by Janssen et al. [14] who observed positive OCB not only in AD but also in other dementias, including frontotemporal lobar degeneration (FTLD) and Creutzfeldt-Jakob disease. In motor neuron diseases (MND), elevated levels of CSF total protein, $Q_{\mathrm{alb}}$, and the presence of intrathecally IgG synthesis were observed $[3$, 31].

Because of these scarce and inconsistent data, the goal of this study was to analyze routine CSF parameters of a large cohort of patients with different neurodegenerative diseases to define a normal reference spectrum of routine findings.

\section{Materials and methods}

\section{Patients}

Data from 765 patients collected by the neurochemicalneuroimmunological laboratory, University of Ulm (Germany) over 10 years (2000-2010) were respectively analyzed and included patients with clinically definite PDD, PD, MSA with subgroups of the parkinsonian type (MSA-P) and the cerebellar type (MSA-C), PSP, corticobasal degeneration (CBD), spinocerebellar ataxia (SCA), MND (including ALS, hereditary spastic paralysis (HSP), and spinal muscular atrophy (SMA)), AD, VD, FTLD, Huntington's disease (HD), patients with muscular diseases (MD) and control subjects with other (neurological) disorders without signs of neurodegenerative diseases $(\mathrm{CON}$, including the diagnoses depression, dissociative disorders, seizures, headache, polyneuropathy, transitoric ischemic attack, and neuritis vestibularis).

CSF lumbar puncture was performed in all patients as part of the routine neurological diagnostics, and all patients have given their written informed consent.

The study was reviewed by the appropriate ethics committee in Ulm (approval number: 20/10) and was performed in accordance with the ethical standards laid down in the 1964 Declaration of Helsinki.

An overview of all CSF data of the 765 patients is given in Table 1.

\section{Analytical procedures}

CSF was collected in polypropylene tubes and was immediately analyzed regarding routine parameters within 1 hour after lumbar puncture. Determination of leukocyte count was performed microscopically in a Fuchs-Rosenthal chamber [9]. CSF samples with contamination of erythrocytes were excluded in order to avoid false positive values of CSF albumin and lactate. Total protein, albumin, $\mathrm{IgG}$, IgA, and IgM were measured by standard immunochemical nephelometry in CSF and serum (Dade-Behring nephelometer analyzer, Marburg, Germany) using a polyclonal antibody in the case of albumin and IgG as well as a latex particle-amplified antibody reaction in the cases of $\operatorname{IgA}$ and IgM. The inter- and intra-assay variabilities were $<10 \%$ for the method-dependent absolute levels as well as for the method-independent CSF/serum quotients of albumin, IgG, IgA, and IgM [25]. CSF lactate was determined by a lactate-oxidase reaction (Greiner $\mathrm{GmbH}$, Flacht, Germany) and detection of oligoclonal bands was performed by isoelectric focusing on agarose gels and subsequent immunoblotting using an IgG-specific antibody staining (Servalyte, Serva) [15]. In demented patients, concentrations of tau and Abeta 1-42 were investigated. Concentrations of CSF tau were determined using an ELISA (Innotest hTAU-Ag; Innogenetics NV, Gent, Belgium) constructed to measure both normal tau and phosphorylated tau (detection limit: $60 \mathrm{pg} / \mathrm{ml}$ ) [35]. The concentration of CSF Abeta 1-42 was determined by an ELISA (Innotest Abeta 1-42; Innogenetics, Belgium) specific for Abeta 1-42 (detection limit: $50 \mathrm{pg} / \mathrm{ml}$ ) [1]. All samples were run in duplicates and ELISAs were performed as earlier described [18].

\section{Evaluation of CSF parameters}

Intrathecal synthesis of $\operatorname{IgG}, \operatorname{IgA}$, and $\operatorname{IgM}$ was detected based on the method of Reiber referring the $\operatorname{IgG}, \operatorname{IgA}$, and IgM quotients to $Q_{\mathrm{alb}}$, with a nonlinear reference range of blood-derived proteins in CSF (see Fig. 1) based on the 
Table 1 Illustration of additional laboratory parameters, including CSF white cell count, CSF lactate, CSF tau and Abeta (A $\beta$ ) 1-42, isolated oligoclonal IgG bands (OCB) in CSF, as well as the diagnostic criteria (DC) for all groups investigated

\begin{tabular}{|c|c|c|c|c|c|c|c|c|c|}
\hline Disease & $N$ & Age \pm SD & Age related $Q_{\mathrm{alb}}$ & Leukocyte count $/ \mu 1$ & Lactate & OCB & Tau in $\mathrm{pg} / \mathrm{ml}$ & $\mathrm{A} \beta$ in $\mathrm{pg} / \mathrm{ml}$ & $\mathrm{DC}$ \\
\hline PDD & 25 & $71 \pm 10$ & 8.7 & $0.64 \pm 0.76$ & $1.72 \pm 0.36$ & 0 & $272 \pm 172$ & $725 \pm 287$ & [7] \\
\hline PD & 47 & $66 \pm 11$ & 8.4 & $0.89 \pm 1.45$ & $1.73 \pm 0.34$ & 0 & $296 \pm 290$ & $967 \pm 312$ & {$[20]$} \\
\hline MSA-P & 12 & $64 \pm 9$ & 8.3 & $1.83 \pm 3.18$ & $1.58 \pm 0.13$ & 0 & $239 \pm 101$ & $755 \pm 105$ & {$[10]$} \\
\hline MSA-C & 13 & $65 \pm 9$ & 8.3 & $1.15 \pm 1.21$ & $1.72 \pm 0.33$ & 0 & $221 \pm 74$ & $794 \pm 250$ & {$[10]$} \\
\hline PSP & 16 & $69 \pm 7$ & 8.6 & $0.75 \pm 1.32$ & $1.79 \pm 0.35$ & 0 & $244 \pm 133$ & $636 \pm 305$ & {$[19]$} \\
\hline CBD & 5 & $69 \pm 9$ & 8.6 & $0.80 \pm 0.84$ & $1.59 \pm 0.17$ & 0 & $221 \pm 91$ & $904 \pm 282$ & {$[6]$} \\
\hline SCA & 6 & $61 \pm 12$ & 8.1 & $0.83 \pm 0.41$ & $1.72 \pm 0.29$ & 0 & $208 \pm 79$ & $916 \pm 214$ & {$[16]$} \\
\hline MND & 177 & $62 \pm 14$ & 8.1 & $1.02 \pm 1.13$ & $1.75 \pm 0.23$ & 1 & $289 \pm 259$ & $924 \pm 305$ & {$[32]$} \\
\hline $\mathrm{AD}$ & 289 & $72 \pm 8$ & 8.8 & $0.73 \pm 2.10$ & $1.76 \pm 0.31$ & 3 & $667 \pm 405$ & $534 \pm 277$ & {$[21]$} \\
\hline FTLD & 28 & $68 \pm 9$ & 8.5 & $0.50 \pm 0.74$ & $1.73 \pm 0.23$ & 0 & $467 \pm 318$ & $672 \pm 233$ & {$[22]$} \\
\hline VD & 13 & $71 \pm 8$ & 8.7 & $1.00 \pm 0.71$ & $1.84 \pm 0.14$ & 0 & $275 \pm 153$ & $683 \pm 162$ & {$[36]$} \\
\hline HD & 31 & $55 \pm 18$ & 7.7 & $1.19 \pm 1.70$ & $1.59 \pm 0.39$ & 0 & - & - & {$[13]$} \\
\hline MD & 20 & $57 \pm 20$ & 7.8 & $3.00 \pm 9.50$ & $1.70 \pm 0.60$ & 1 & - & - & - \\
\hline $\mathrm{CON}$ & 83 & $53 \pm 18$ & 7.5 & $1.28 \pm 2.92$ & $1.73 \pm 0.34$ & 0 & - & - & - \\
\hline
\end{tabular}

$P D D$ Parkinson disease dementia, $P D$ Parkinson disease, $M S A-P / C$ multisystem atrophy with Parkinson/cerebellar syndrome, $P S P$ progressive supranuclear palsy, $C B D$ corticobasal degeneration, $S C A$ spinocerebellar atrophy, MND motorneuron disease, AD Alzheimer disease, FTLD frontotemporal lobar degeneration, $V D$ vascular dementia, $H D$ Huntington disease, $M D$ muscle disease, $C O N$ control group

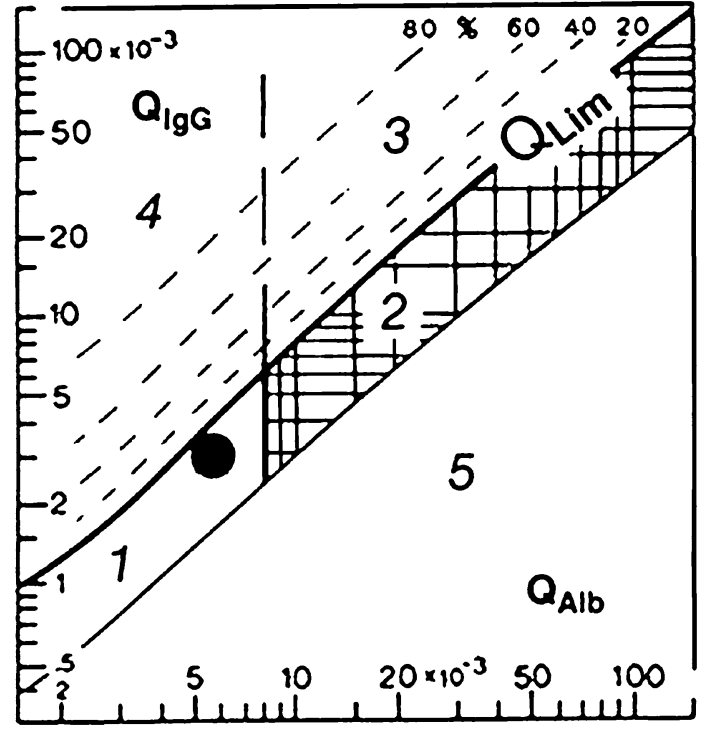

Fig. 1 CSF-serum quotient diagram for IgG with hyperbolic reference range according to Reiber [24]. On the abscissa, a $Q_{\text {alb }}$ of 1 represents a normal blood-CSF barrier function; 2 demonstrated a blood-CSF barrier dysfunction. The ordinate demonstrates $Q_{\mathrm{IgG}}$ with the upper limit for $Q_{\text {lim }}, Q_{\text {mean }}$, and $Q_{\text {low }}$, whereby values below $Q_{\text {lim }}$ can discriminate the blood-derived IgG fraction from an intrathecal Ig synthesis either represented by 4 as intrathecally IgG synthesis only or as 3 demonstrating a combined blood-CSF barrier function together with an intrathecal IgG-synthesis. 5 is not possible and indicates a methodological error in the measurement

physiologically and theoretically derived hyperbolic functions $[24,26]$. The data are presented to the clinician either graphically in the diagrams by Reiber, so called Reibergrams (see Fig. 3) or numerically by calculation of the intrathecally synthesized amount $\left(\operatorname{Ig}_{\text {loc }}\right)$ in $\mathrm{mg} / \mathrm{l} \mathrm{CSF}$ or as the relative intrathecal fraction $\left(\mathrm{Ig}_{\mathrm{IF}}\right)$ in $\%$ of the total $\mathrm{Ig}$ CSF concentration. To ensure maximal sensitivity, the interpretation of an intrathecal synthesis refers to the upper border line of the reference range $\left(Q_{\lim }\right.$ in the quotient diagrams). This line is based on $Q_{\text {mean }}+3$ s (or a reference range including $99 \%$ of the noninflammatory cases). The comparison of different patient groups in the nonlinear evaluation diagrams needs the reference to the mean values of the reference range in the Reibergrams $\left(Q_{\text {mean }}\right)$ as evaluated in the CSF statistics tool ("Protein Statistics in CSF Analysis with Reibergrams", Version 3.0, COMED). Intrathecal $\mathrm{Ig}$ synthesis is calculated with $\mathrm{Ig}_{\text {loc }}$ in $\mathrm{mg} / \mathrm{l}$ replacing $Q_{\text {lim }}$ by $Q_{\text {mean }}$. $\mathrm{Ig}_{\mathrm{IF}}$, the relative intrathecal fraction is not useful for statistics of groups.Intrathecal Ig synthesis with reference to $Q_{\text {mean }}$ is calculated according to:

$\operatorname{Ig}_{\mathrm{loc}}($ mean $)=\left(Q_{\mathrm{Ig}}-Q_{\mathrm{Ig}}(\right.$ mean $\left.)\right) \times \operatorname{Ig}$ serum $[\mathrm{mg} / \mathrm{l}]$.

\section{Statistics}

Assessment of the mean quantity and frequency of intrathecal Ig synthesis, frequency of blood-CSF barrier dysfunction and Reibergrams were determined using the software "Protein Statistics in CSF Analysis with Reibergrams" (Version 3.0, COMED), which calculates quotients and the intrathecally synthesized fractions $\left[\operatorname{Ig} X_{\text {loc }}(\mathrm{m})\right.$ or $\left.\operatorname{IgX} \mathrm{IF}_{\mathrm{IF}}(\mathrm{m})\right]$ with reference to the $Q_{\text {mean }}$ of the reference range. The age-related reference range of $Q_{\text {alb }}$ was calculated as $Q_{\mathrm{alb}}=\left(4+\right.$ age/15) $\times 10^{-3}$ [29]. The calculated 
upper limit of the reference range of $Q_{\mathrm{alb}}$ is also a function of the volume of CSF extraction. To allow a modification of the decision cutoff in cases of unknown volumes of extraction, we refer to two different levels, a $10 \%$ and a $20 \%$ level. The $20 \%$ level $\left[Q_{\text {alb }}=(4+\right.$ age $/ 15) \times$ $\left.10^{-3} \times 1.2\right]$ is best for small extraction volumes to increase the reliability of an interpretation as a blood-CSF barrier dysfunction; thus, the statistical analysis was performed on that basis because the exact CSF volumes of all samples could not retrospectively be identified.

To test the significance of the difference between the calculated means and the mean of the noninflammatory control group, we used the program available at http:// daten-consult.de/forms/ttestunabh.html. The control group is represented by 4,300 patients without a humoral immune response in the CSF [24]. The investigation of the smaller $\mathrm{CON}$ group in our laboratory was performed as an internal control, but all statistical analyzes are related to the control group of reference [24], which is the basis of statistical examinations in the software program. $P$ values below 0.05 are considered significant.

\section{Results}

Frequency and extent of intrathecal Ig synthesis and $\mathrm{OCB}$

In $\mathrm{AD}$, intrathecal Ig synthesis of $\operatorname{IgA}$ and $\operatorname{IgM}$ were found in 2 and 3 cases, respectively, with positive OCB. Additional analyses including PCR and antibody indices (AI) for Borrelia burgdorferi showed negative results and because $\operatorname{Ig}_{\text {loc }}$ (mean) was also negative, statistical analyses were not performed. Intrathecal IgM synthesis in 2 patients with PDD was accompanied by negative OCB and normal white cell counts. Thus, because the $\operatorname{Ig}_{\text {loc }}$ (mean) was positive, statistical analysis was performed with a nonsignificant $p$ value of 0.851 . In 2 MND patients having intrathecal Ig synthesis of $\mathrm{IgG}$ and $\mathrm{IgA}$, respectively, accompanied by positive $\mathrm{OCB}, \mathrm{Ig}_{\text {loc }}$ (mean) was negative; thus, statistical analysis was renounced. As $\mathrm{Ig}_{\text {loc }}$ (mean) was negative for $\operatorname{IgG}, \operatorname{IgA}$, and $\operatorname{IgM}$ in the other patient groups, the calculation of mean values of $\operatorname{Ig} \mathrm{X}_{\mathrm{IF}}(\mathrm{m})$ was not reasonable due to the fact that negative values are not possible by definition and a statistical evaluation is not possible in cases of a negative $\operatorname{IgX} \mathrm{X}_{\mathrm{loc}}(\mathrm{m})$. For detailed information see Online Resource Table 1.

Frequencies of blood-CSF barrier dysfunction

In relation to the age-matched $Q_{\mathrm{alb}}$, elevated $Q_{\mathrm{alb}}$ values with an increase of $\geq 25 \%$ were detected in PSP and MSA$P$ in $23 \%$ of the patients in VD, in $20 \%$ of the patients in
PDD, and in $46 \%$ of the cases of MSA-C (see Online Resource Table 2).

Outliers of CSF-blood barrier dysfunction

Concerning dysfunction of the CSF-blood barrier, reasons for all outliers were detected. Here, osteodegenerative diseases with stenosis of the spinal canal could often traced back to the herniation of a lumbar intervertebral disk or to a scoliosis as a frequent cause for elevated $Q_{\text {alb values (as }}$ seen in 1 PD patient, 1 patient among the persons with MSA-P, 2 patients in the MSA-C group, 2 AD patients as well as in $1 \mathrm{PDD}$ patient, $1 \mathrm{HD}$ diseased person, $1 \mathrm{MD}$ patient, and 1 patient of the CON group). Besides degenerative processes of the spine, metabolic dysfunctions related to insufficiently treated diabetes with severe diabetic vasculo- and neuropathy were additional causes for the elevated $Q_{\text {Alb }}$ values as detected in 1 FTLD patient. For detailed information, see Fig. 2 and Online Resource Table 2 .

Lactate levels and white cell count

Reference values of L-lactate are age-dependent (mmol/l $=\mathrm{mg} / \mathrm{dl} \times 0.11)$ :

0-15 years: $1.1-1.8 \mathrm{mmol} / \mathrm{l}(9.9-16.2 \mathrm{mg} / \mathrm{dl})$

16-50 years: $1.5-2.1 \mathrm{mmol} / \mathrm{l}(13.5-18.9 \mathrm{mg} / \mathrm{dl})$

>51 years: $1.7-2.6 \mathrm{mml} / \mathrm{l}(15.3-23.4 \mathrm{mg} / \mathrm{dl})$

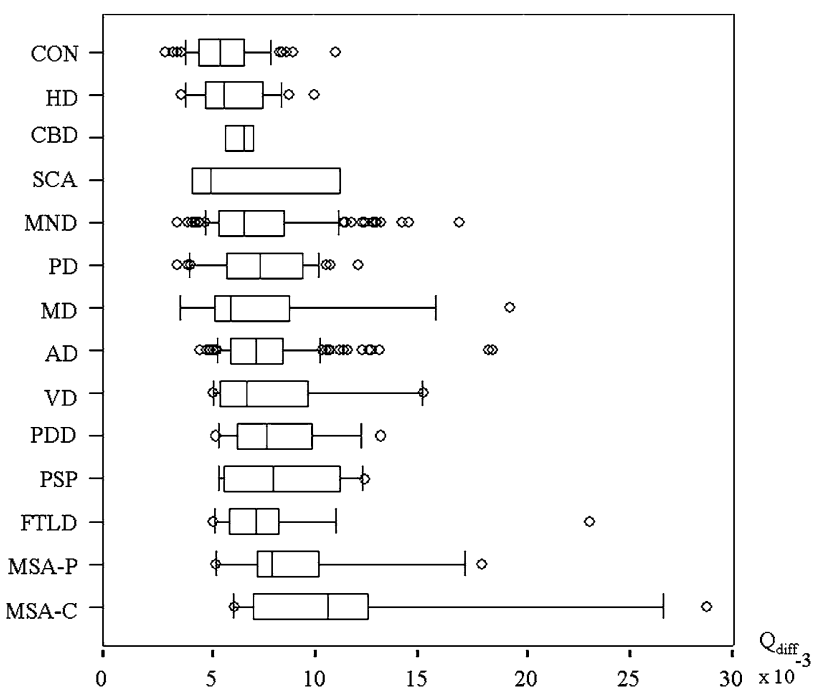

Fig. 2 Illustration of age-related $Q_{\text {alb. }}$ CSF-blood barrier (dys-) function of all groups are indicated on the basis of $Q_{\text {diff }}$ that represents the age-dependant CSF-blood barrier function according to $Q_{\text {diff }}=$ $Q_{\text {alb }}-Q_{\mathrm{alb}} *\left(\right.$ with $Q_{\mathrm{alb}} *=(4+$ age in years $\left.\left.) / 15\right) \times 10^{-3}\right)$. The $b o x$ represents the 25 th to 75 th quartile, whiskers represent the range, the vertical line in the boxes shows the median and circles depict outliers. The numbers of patients correspond to the numbers given in Table 1 
No elevated lactate levels were detected in any of the groups investigated. Regarding leukocyte counts, 1 patient of the MD group had an increased level of 43 leukocytes/ $\mu 1$ traced back to a reactive pleocytosis in the scope of septicemia by phlegmon.

Data are summarized in Table 1.

\section{Reibergrams}

The CSF-serum albumin concentration quotient is generally accepted as the best indicator to characterize the blood-CSF barrier function for blood-derived proteins in the CSF; this is due to the fact that albumin in CSF exclusively originates from blood. In case of pathological changes, $Q_{\mathrm{alb}}$ is the most relevant parameter to indicate a change in blood-CSF barrier function. This quality of $Q_{\text {alb }}$ led to the development of quotient diagrams in which the immunoglobulin quotients are referred to the albumin quotient, a model that allows quantification of an intrathecal protein fraction independent of the individual bloodbarrier function. Former methods to measure the intrathecal IgG synthesis according to Delpech-Lichtblau (IgG index) or Tourtellotte are based on a linear CSF-blood flow and are associated with false-positive values for intrathecal IgG (with higher errors for IgA and $\operatorname{IgM}$ ) in cases of high $Q_{\text {alb }}$. The model of Reiber [24] for the blood-CSF barrier function describes a diffusion system where on one side of a restricted range (tissue) is the solute (blood) and at the other side a solvent (CSF) which is the source of a steady elimination process by the flowing CSF in a hyperbolic, nonlinear range.

The graphic representation of the Reibergrams allows a direct visual interpretation of the data (see Fig. 1). Values above $Q_{\text {lim }}$ (for the individual patient) or $Q_{\text {mean }}$ (for a group of patients), respectively, indicate the intrathecal fraction of a protein (IgG, IgA, and IgM) in $\%$ (whereby $Q_{\text {lim }} / Q_{\text {mean }}$ represent $0 \%$ ). The limit for $Q_{\mathrm{alb}}$ as a measure for the CSF-blood barrier function is age-dependant according to $Q_{\text {alb }}=(4+$ age in years $\left.) / 15\right) \times 10^{-3}$.

Figure 3 illustrates the Reibergrams that use the associated $\operatorname{IgG}, \operatorname{IgA}$, and $\operatorname{IgM}$ diagrams depicted horizontally for better pattern recognition among the different patient groups, especially when the variability of $Q_{\mathrm{alb}}$ is considered.

\section{Discussion}

CSF routine parameters are an integral part of the diagnostic procedure in neurodegenerative diseases and form the basis for further disease-specific examinations. For this reason, they have been included in the guidelines for dementia diagnostics by the German societies for psychiatry and neurology [5]. To give an overview of what comprises the normal reference spectrum, to decide in which cases examination of special parameters is indicated, and to consider when it is necessary to re-evaluate the preliminary diagnosis, and reflect on possible alternative mechanisms as a reason for the pathological values, we retrospectively analyzed routine CSF data collected in our neurochemistry laboratory.

\section{Intrathecal Ig synthesis and OCB}

In contrast to chronic inflammatory diseases of the CNS [28], we observed mild intrathecal Ig synthesis in only a few cases, which could be either traced back to a former infection of the CNS or to an immune process in a subfraction of patients with neurodegenerative diseases [11]. Because leukocyte counts and lactate levels were normal in those cases, we favor the explanation of an immunological scar due to a former infection. Highly elevated Ig values as indicators of an inflammatory process are not representative and should lead to a re-evaluation of the data by repeated investigations to exclude false-positive measurements and in cases of confirmed elevated values, to the investigation of special parameters (PCR or antigen specific antibodies). This is important for those cases where an early infection of the CNS is presented by intrathecal Ig synthesis with negative OCB $[4,12]$ and should lead to reevaluation of the neurodegenerative diagnosis, whereby the seldom simultaneously occurrence of a neurodegenerative and an inflammatory disease has to be taken into account.

\section{Blood-CSF barrier dysfunction}

Dysfunction of the CSF-blood barrier is present in various neurological diseases of inflammatory and noninflammatory etiology and may even be found in patients with no neurological diseases [3]. We and others [17] observed elevated frequencies of $Q_{\text {alb }}$ between 25 and $45 \%$ in PSP and MSA, possibly due back to an age-related effect with a reduction of the CSF flow, an increased prevalence of osteodegenerative diseases of the spine, or metabolic dysfunctions, such as diabetes or obesity. In accordance with previous findings [3, 11], we found CSF-blood barrier dysfunctions with frequencies of 20-25\% in PDD and VD, whereby dysfunction of endothelial cells with increased permeability of serum proteins was hypothesized in VD as a pathophysiological explanation [30]. Regarding the subgroup of MND patients, we and others found elevated levels of CSF total protein associated with a slightly impaired CSF-blood barrier dysfunction [2, 31]. In GBS and CIDP, reduction of the CSF flow rate traced back to an inflammatory swelling in the region of the spinal roots as possibly being a reason for the $Q_{\mathrm{Alb}}$ elevation [34]. Similar 

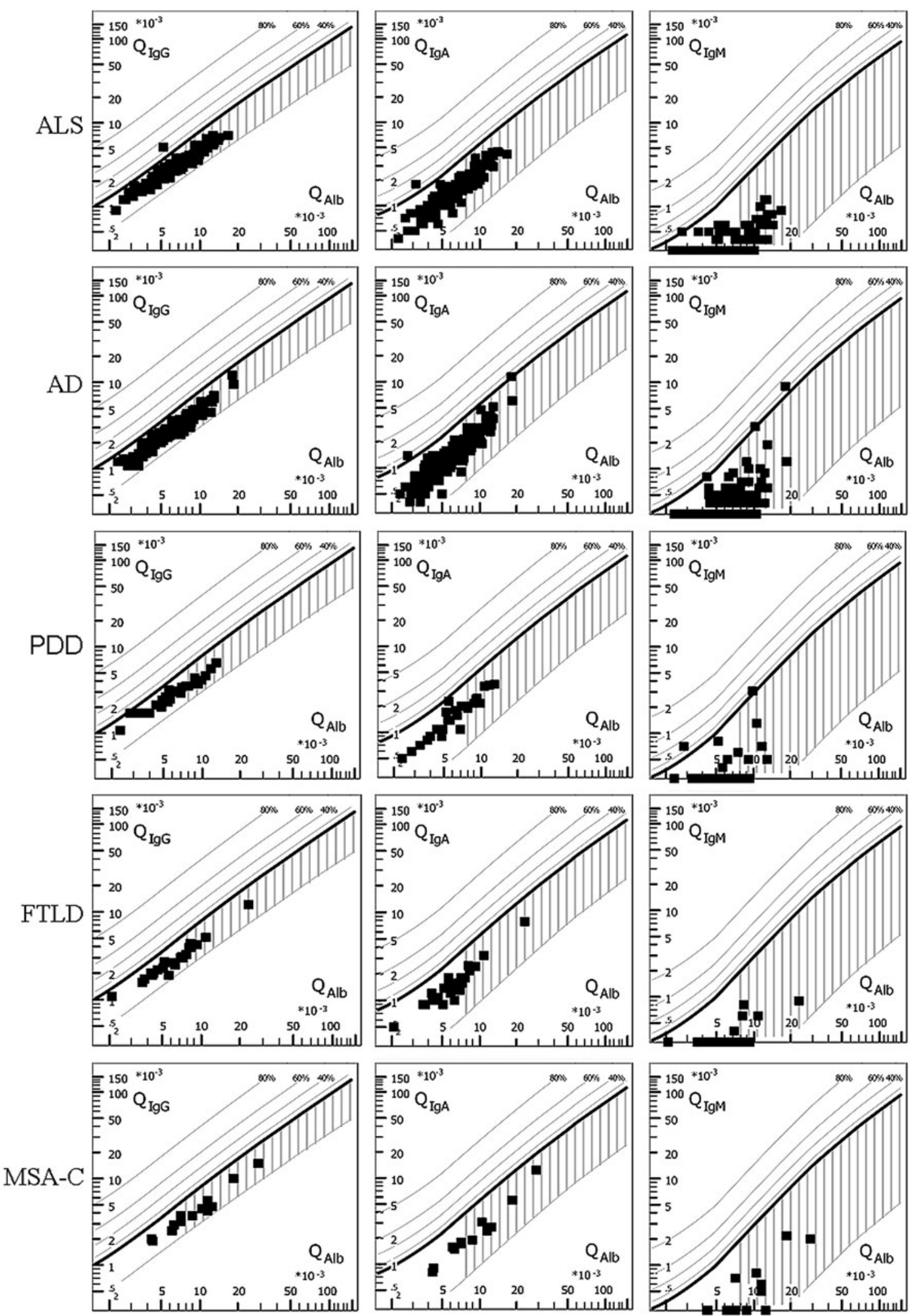

Fig. 3 Illustration of Reibergrams for all patient groups. Q-IgG, Q-IgA, and Q-IgM are indicated in relation to the CSF-blood barrier function, represented by $Q_{\mathrm{alb}}$. For interpretation of the Reibergrams, see Fig. 1 
pathophysiological processes in MND could be responsible for CSF-blood barrier dysfunction, where structural alterations of the anterior nerve roots may be responsible for reduction of CSF - flow rate. So that in all cases with highly elevated $Q_{\mathrm{Alb}}$, alternative reasons should be excluded, e.g., metabolic as well as myelostenotic processes being the most frequent.

White cell count and lactate levels

Among the patients investigated, only 1 case with MD showed elevated white cell count due to a coincidental systemic infection, so that elevation of leukocyte count and lactate levels are primarily not compatible with the diagnosis of neurodegenerative diseases and should lead to investigation of alternative reasons. In addition, an increase of both parameters may be helpful in cases with intrathecal Ig synthesis to exclude or confirm inflammatory processes and may support the decision whether additional CSF parameters should be investigated.

Comprehensive, CSF routine parameters are necessary to exclude other nonneurodegenerative diseases and should be introduced into neurochemical laboratories. Slight elevation of $Q_{\text {Alb }}$ and mild intrathecal Ig synthesis seem to be nonspecific findings in these disorders and do not exclude the diagnosis. However, highly pathological values of routine parameters were not observed and are suggestive for other diseases or alternative mechanisms if a neurodegenerative disease is still supposed.

Acknowledgments Special thanks go to Refika Aksamija, Dr. Vera Lehmensiek, Rehaneh Mojib, Dagmar Vogel, and Alice Pabst for not only measurement of the routine and specific parameters, but also for organizing and cataloging patient data. This study was supported in part by grant P-LS-Prot/42 from the Landesstiftung Baden-Württemberg (Drs. Jesse and Otto), by grants NADINE, cNeupro and NeuroTAS from the European Commission (Dr. Otto), and the Heidelberger Akademie der Wissenschaften (Dr. von Arnim).

Open Access This article is distributed under the terms of the Creative Commons Attribution Noncommercial License which permits any noncommercial use, distribution, and reproduction in any medium, provided the original author(s) and source are credited.

\section{References}

1. Andreasen N, Hesse C, Davidsson P, Minthon L, Wallin A, Winblad B, Vanderstichele H, Vanmechelen E, Blennow $\mathrm{K}$ (1999) Cerebrospinal fluid beta-amyloid(1-42) in Alzheimer disease: differences between early- and late-onset Alzheimer disease and stability during the course of disease. Arch Neurol 56:673-680

2. Apostolski S et al (1991) Serum and CSF immunological findings in ALS. Acta Neurol Scand 83(2):96-98
3. Brettschneider J, Claus A, Kassubek J, Tumani H (2005) Isolated blood-cerebrospinal fluid barrier dysfunction: prevalence and associated diseases. J Neurol 252:1067-1073

4. Brettschneider J, Tumani H, Kiechle U, Muche R, Richards G, Lehmensiek V, Ludolph AC, Otto M (2009) IgG antibodies against measles, rubella, and varicella zoster virus predict conversion to multiple sclerosis in clinically isolated syndrome. PLoS One 4:e7638

5. Deutsche Gesellschaft für Psychiatrie PuN, Deutsche Gesellschaft für Neurologie (2009) S3-Leitlinie Demenzen. 038/013

6. Dickson DW, Bergeron C, Chin SS, Duyckaerts C, Horoupian D, Ikeda K, Jellinger K, Lantos PL, Lippa CF, Mirra SS, Tabaton M, Vonsattel JP, Wakabayashi K, Litvan I (2002) Office of rare diseases neuropathologic criteria for corticobasal degeneration. J Neuropathol Exp Neurol 61:935-946

7. Dubois B, Burn D, Goetz C, Aarsland D, Brown RG, Broe GA, Dickson D, Duyckaerts C, Cummings J, Gauthier S, Korczyn A, Lees A, Levy R, Litvan I, Mizuno Y, McKeith IG, Olanow CW, Poewe W, Sampaio C, Tolosa E, Emre M (2007) Diagnostic procedures for Parkinson's disease dementia: recommendations from the movement disorder society task force. Mov Disord 22:2314-2324

8. Frolich L, Kornhuber J, Ihl R, Fritze J, Maurer K, Riederer P (1991) Integrity of the blood-CSF barrier in dementia of Alzheimer type: CSF/serum ratios of albumin and IgG. Eur Arch Psychiatry Clin Neurosci 240:363-366

9. Fuchs A, Rosenthal R (1904) Physikalisch-chemische, zytologische und anderweitige Untersuchungen der Cerebrospinalflüssigkeit. Wien Med Presse 45:2081-2087

10. Gilman S, Low P, Quinn N, Albanese A, Ben-Shlomo Y, Fowler C, Kaufmann H, Klockgether T, Lang A, Lantos P, Litvan I, Mathias C, Oliver E, Robertson D, Schatz I, Wenning G (1998) Consensus statement on the diagnosis of multiple system atrophy. American Autonomic Society and American Academy of Neurology. Clin Auton Res 8:359-362

11. H Hampel, Müller-Spahn F, Berger C, Haberl A, Ackenheil M, Hock C (1995) Evidence of blood-cerebrospinal fluid-barrier impairment in a subgroup of patients with dementia of the Alzheimer type and major depression: a possible indicator for immunoactivation. Dementia 6:348-354

12. Hampel H, Kotter HU, Padberg F, Korschenhausen DA, Moller HJ (1999) Oligoclonal bands and blood-cerebrospinal-fluid barrier dysfunction in a subset of patients with Alzheimer disease: comparison with vascular dementia, major depression, and multiple sclerosis. Alzheimer Dis Assoc Disord 13:9-19

13. Harbo HF, Finsterer J, Baets J, Van Broeckhoven C, Di Donato S, Fontaine B, De Jonghe P, Lossos A, Lynch T, Mariotti C, Schols L, Spinazzola A, Szolnoki Z, Tabrizi SJ, Tallaksen C, Zeviani M, Burgunder JM, Gasser T (2009) EFNS guidelines on the molecular diagnosis of neurogenetic disorders: general issues, Huntington's disease, Parkinson's disease and dystonias. Eur J Neurol 16:777-785

14. Janssen JC, Godbolt AK, Ioannidis P, Thompson EJ, Rossor MN (2004) The prevalence of oligoclonal bands in the CSF of patients with primary neurodegenerative dementia. J Neurol 251:184-188

15. Keir G, Luxton RW, Thompson L (1990) Isoelectric focusing of cerebrospinal fluid immunoglobulin G: an annotated update. Ann Clin Biochem 27:436-443

16. Klockgether $\mathrm{T}$ (2005) Ataxias Diagnostic procedure and treatment. Nervenarzt 76:1275-1283 quiz 1284-1275

17. Leonardi A, Abbruzzese G, Arata L, Cocito L, Vische M (1984) Cerebrospinal fluid (CSF) findings in amyotrophic lateral sclerosis. J Neurol 231:75-78

18. Lewczuk P, Esselmann H, Bibl M, Beck G, Maler JM, Otto M, Kornhuber J, Wiltfang J (2004) Tau protein phosphorylated at threonine 181 in CSF as a neurochemical biomarker in 
Alzheimer's disease: original data and review of the literature. J Mol Neurosci 23:115-122

19. Litvan I, Agid Y, Calne D, Campbell G, Dubois B, Duvoisin RC, Goetz CG, Golbe LI, Grafman J, Growdon JH, Hallett M, Jankovic J, Quinn NP, Tolosa E, Zee DS (1996) Clinical research criteria for the diagnosis of progressive supranuclear palsy (Steele-Richardson-Olszewski syndrome): report of the NINDSSPSP international workshop. Neurology 47:1-9

20. McKeith IG (2006) Consensus guidelines for the clinical and pathologic diagnosis of dementia with Lewy bodies (DLB): report of the consortium on DLB international workshop. J Alzheimers Dis 9:417-423

21. McKhann G, Drachman D, Folstein M, Katzman R, Price D, Stadlan EM (1984) Clinical diagnosis of Alzheimer's disease: report of the NINCDS-ADRDA work group under the auspices of Department of Health and Human Services Task Force on Alzheimer's Disease. Neurology 34:939-944

22. Neary D, Snowden JS, Gustafson L, Passant U, Stuss D, Black S, Freedman M, Kertesz A, Robert PH, Albert M, Boone K, Miller BL, Cummings J, Benson DF (1998) Frontotemporal lobar degeneration: a consensus on clinical diagnostic criteria. Neurology 51:1546-1554

23. Otto M (2010) Dementia diagnostics 2.0-transfer from research studies into routine clinical practice. Clin Chem 56:152-153

24. Reiber H (1994) Flow rate of cerebrospinal fluid (CSF)- a concept common to normal blood-CSF barrier function and to dysfunction in neurological diseases. J Neurol Sci 122:189-203

25. Reiber H (1995) External quality assessment in clinical neurochemistry: survey of analysis for cerebrospinal fluid (CSF) proteins based on CSF/serum quotients. Clin Chem 41:256-263

26. Reiber H (2003) Proteins in cerebrospinal fluid and blood: barriers, CSF flow rate and source-related dynamics. Restor Neurol Neurosci 21:79-96

27. Reiber H, Peter JB (2001) Cerebrospinal fluid analysis-diseaserelated data patterns and evaluation programs. J Neurol Sci 184:101-122
28. Reiber H, Ungefehr S, Jacobi C (1998) The intrathecal, polyspecific and oligoclonal immune response in multiple sclerosis. Mult Scler 4:111-117

29. Reiber H, Otto M, Trendelenburg C, Wormek A (2001) Reporting cerebrospinal fluid data: knowledge base and interpretation software. Clin Chem Lab Med 39:324-332

30. Skoog I, Wallin A, Fredman P, Hesse C, Aevarsson O, Karlsson I, Gottfries CG, Blennow K (1998) A population study on bloodbrain barrier function in 85-year-olds: relation to Alzheimer's disease and vascular dementia. Neurology 50:966-971

31. Süssmuth SD (2008) Biochemical markers in CSF of ALS patients. Curr Med Chem 15:1788-1801

32. The EFNS Task Force on Diagnosis, Management of Amyotrophic Lateral Sclerosis (2005) Guidelines for diagnosing and clinical care of patients and relatives. Eur J Neurol 12:921-938

33. Tumani H, Teunissen C, Sussmuth S, Otto M, Ludolph AC, Brettschneider J (2008) Cerebrospinal fluid biomarkers of neurodegeneration in chronic neurological diseases. Expert Rev Mol Diagn 8:479-494

34. Upton ML, Weller RO (1985) The morphology of cerebrospinal fluid drainage pathways in human arachnoid granulations. J Neurosurg 63:867-875

35. Vandermeeren M, Mercken M, Vanmechelen E, Six J, van de Voorde A, Martin JJ, Cras P (1993) Detection of tau proteins in normal and Alzheimer's disease cerebrospinal fluid with a sensitive sandwich enzyme-linked immunosorbent assay. J Neurochem 61:1828-1834

36. Waldemar G, Dubois B, Emre M, Georges J, McKeith IG, Rossor M, Scheltens P, Tariska P, Winblad B (2007) Recommendations for the diagnosis and management of Alzheimer's disease and other disorders associated with dementia: EFNS guideline. Eur J Neurol 14:e1-26

37. Zimmermann R, Beck G, Knispel S, Maler JM, Weih M, Wiltfang J, Kornhuber J, Lewczuk P (2010) Intrathecal IgG synthesis in patients with alterations in the neurochemical dementia diagnostics. J Alzheimers Dis 19:1199-1203 